\title{
NuSTAR and XMM-Newton observations of NGC 1365: Extreme absorption variability and a constant inner accretion disk
}

Walton, D. J.; Risaliti, G.; Harrison, F. A.; Fabian, A. C.; Miller, J. M.; Arevalo, P.; Ballantyne, D. R.; Boggs, S. E.; Brenneman, L. W.; Christensen, Finn Erland

Total number of authors:

27

Published in:

Astrophysical Journal

Link to article, DOI:

10.1088/0004-637X/788/1/76

Publication date:

2014

Document Version

Publisher's PDF, also known as Version of record

Link back to DTU Orbit

Citation $(A P A)$ :

Walton, D. J., Risaliti, G., Harrison, F. A., Fabian, A. C., Miller, J. M., Arevalo, P., Ballantyne, D. R., Boggs, S. E., Brenneman, L. W., Christensen, F. E., Craig, W. W., Elvis, M., Fuerst, F., Gandhi, P., Grefenstette, B. W., Hailey, C. J., Kara, E., Luo, B., Madsen, K. K., ... Zhang, W. W. (2014). NuSTAR and XMM-Newton observations of NGC 1365: Extreme absorption variability and a constant inner accretion disk. Astrophysical Journal, 788(1), [76]. https://doi.org/10.1088/0004-637X/788/1/76

\section{General rights}

Copyright and moral rights for the publications made accessible in the public portal are retained by the authors and/or other copyright owners and it is a condition of accessing publications that users recognise and abide by the legal requirements associated with these rights.

- Users may download and print one copy of any publication from the public portal for the purpose of private study or research.

- You may not further distribute the material or use it for any profit-making activity or commercial gain

- You may freely distribute the URL identifying the publication in the public portal 


\title{
NUSTAR AND XMM-NEWTON OBSERVATIONS OF NGC 1365: EXTREME ABSORPTION VARIABILITY AND A CONSTANT INNER ACCRETION DISK
}

\author{
D. J. Walton ${ }^{1}$, G. Risaliti ${ }^{2,3}$, F. A. Harrison ${ }^{1}$, A. C. Fabian $^{4}$, J. M. Miller ${ }^{5}$, P. Arevalo ${ }^{6}$, D. R. Ballantyne ${ }^{7}$, \\ S. E. Boggs ${ }^{8}$, L. W. Brenneman ${ }^{3}$, F. E. Christensen ${ }^{9}$, W. W. Craig ${ }^{8}$, M. Elvis ${ }^{3}$, F. Fuerst ${ }^{1}$, P. Gandhi ${ }^{10}$, \\ B. W. Grefenstette ${ }^{1}$, C. J. Hailey ${ }^{11}$, E. Kara ${ }^{4}$, B. Luo ${ }^{12,13}$, K. K. Madsen ${ }^{1}$, A. Marinucci ${ }^{14}$, G. Matt ${ }^{14}$, \\ M. L. PARKer ${ }^{4}$, C. S. Reynolds ${ }^{15,16}$, E. Rivers ${ }^{1}$, R. R. Ross ${ }^{17}$, D. Stern ${ }^{18}$, ANd W. W. Zhang ${ }^{19}$ \\ ${ }^{1}$ Cahill Center for Astronomy and Astrophysics, California Institute of Technology, Pasadena, CA 91125, USA \\ ${ }^{2}$ INAF-Osservatoria Astrofisico di Arcetri, Largo Enrico Fermi 5, I-50125 Firenze, Italy \\ ${ }^{3}$ Harvard-Smithsonian Center for Astrophysics, 60 Garden Street, Cambridge, MA 02138, USA \\ ${ }^{4}$ Institute of Astronomy, University of Cambridge, Madingley Road, Cambridge CB3 OHA, UK \\ ${ }^{5}$ Department of Astronomy, University of Michigan, 500 Church Street, Ann Arbor, MI 48109-1042, USA \\ ${ }^{6}$ Pontificia Universidad Católica de Chile, Instituto de Astrfísica, Casilla 306, Santiago 22, Chile \\ ${ }^{7}$ Center for Relativistic Astrophysics, School of Physics, Georgia Institute of Technology, Atlanta, GA 30332, USA \\ ${ }^{8}$ Space Sciences Laboratory, University of California, Berkeley, CA 94720, USA \\ ${ }^{9}$ DTU Space, National Space Institute, Technical University of Denmark, Elektrovej 327, DK-2800 Lyngby, Denmark \\ ${ }^{10}$ Department of Physics, Durham University, South Road, Durham DH1 3LE, UK \\ ${ }^{11}$ Columbia Astrophysics Laboratory, Columbia University, New York, NY 10027, USA \\ 12 Department of Astronomy and Astrophysics, 525 Davey Lab, The Pennsylvania State University, University Park, PA 16802, USA \\ ${ }^{13}$ Institute for Gravitation and the Cosmos, The Pennsylvania State University, University Park, PA 16802, USA \\ ${ }^{14}$ Dipartimento di Matematica e Fisica, Universita degli Studi Roma Tre, via della Vasca Navale 84, I-00146 Roma, Italy \\ ${ }^{15}$ Department of Astronomy, University of Maryland, College Park, MD 20742, USA \\ 16 Joint Space-Science Institute (JSI), University of Maryland, College Park, MD 20742, USA \\ ${ }^{17}$ Physics Department, College of the Holy Cross, Worcester, MA 01610, USA \\ ${ }^{18}$ Jet Propulsion Laboratory, California Institute of Technology, Pasadena, CA 91109, USA \\ ${ }_{19}$ NASA Goddard Space Flight Center, Greenbelt, MD 20771, USA \\ Received 2013 November 27; accepted 2014 April 18; published 2014 May 23
}

\begin{abstract}
We present a spectral analysis of four coordinated NuSTAR+XMM-Newton observations of the Seyfert galaxy NGC 1365. These exhibit an extreme level of spectral variability, which is primarily due to variable line-of-sight absorption, revealing relatively unobscured states in this source for the first time. Despite the diverse range of absorption states, each of the observations displays the same characteristic signatures of relativistic reflection from the inner accretion disk. Through time-resolved spectroscopy, we find that the strength of the relativistic iron line and the Compton reflection hump relative to the intrinsic continuum are well correlated, which is expected if they are two aspects of the same broadband reflection spectrum. We apply self-consistent disk reflection models to these time-resolved spectra in order to constrain the inner disk parameters, allowing for variable, partially covering absorption to account for the vastly different absorption states that were observed. Each of the four observations is treated independently to test the consistency of the results obtained for the black hole spin and the disk inclination, which should not vary on observable timescales. We find both the spin and the inclination determined from the reflection spectrum to be consistent, confirming that NGC 1365 hosts a rapidly rotating black hole; in all cases the dimensionless spin parameter is constrained to be $a^{*}>0.97$ (at $90 \%$ statistical confidence or better).
\end{abstract}

Key words: black hole physics - galaxies: active - X-rays: individual (NGC 1365)

Online-only material: color figures

\section{INTRODUCTION}

Black hole spin is a quantity of significant importance for addressing a variety of astrophysical topics, including the growth of the supermassive black holes powering active galactic nuclei (AGNs; e.g., Dubois et al. 2013), the formation of black hole binaries in supernova explosions (Miller et al. 2011), and potentially the launch of powerful relativistic jets (Blandford \& Znajek 1977), although the exact role spin plays here is still controversial (Steiner et al. 2013; King et al. 2013; Russell et al. 2013). For active galaxies, the best method available for measuring black hole spin is to measure the relativistic distortions of fluorescent line emission from the inner accretion disk, excited through irradiation by hard X-rays. These relativistic effects broaden and skew intrinsically narrow emission lines into a characteristic "diskline" profile (Fabian et al. 1989; Laor 1991), which depends on the black hole spin.
The most prominent features produced by such irradiation are typically the iron $\mathrm{K} \alpha$ emission line at $\sim 6.4-7.0 \mathrm{keV}$ (depending on the ionization state) and a broad peak in the reflected continuum at $\sim 30 \mathrm{keV}$ referred to as the Compton hump (George \& Fabian 1991). Roughly 40\% of X-ray bright AGNs display evidence for broadened iron $\mathrm{K} \alpha$ emission (Nandra et al. 2007; de La Calle Pérez et al. 2010), and the majority of AGNs also show a "hard" excess above $\sim 10 \mathrm{keV}$ (e.g., Nandra \& Pounds 1994; Perola et al. 2002; Dadina 2007; Rivers et al. 2013), consistent with Compton reflection from the accretion disk (e.g., Walton et al. 2010; Nardini et al. 2011). Spin estimates for a growing sample ( $\sim 20-30$ sources) of local AGNs have recently been obtained through study of these features, e.g., Brenneman \& Reynolds (2006), Miniutti et al. (2009), Zoghbi et al. (2010), Brenneman et al. (2011), Nardini et al. (2012), Gallo et al. (2013), Walton et al. (2013b); see also Miller (2007), Reynolds (2013), and Brenneman (2013) for recent reviews. 
Table 1

Basic Observational Details for the Coordinated NuSTAR+XMM-Newton Observations of NGC 1365

\begin{tabular}{llcc}
\hline \hline Observation & Date & \multicolumn{2}{c}{ Total Good Exposures (ks) } \\
\cline { 3 - 4 } & & XMM-Newton ${ }^{\mathrm{a}}$ & NuSTAR $^{\mathrm{b}}$ \\
\hline 1 & 2012 Jul & $110 / 130$ & 77 \\
2 & 2012 Dec & $93 / 120$ & 66 \\
3 & 2013 Jan & $90 / 118$ & 74 \\
4 & 2013 Feb & $103 / 119$ & 70 \\
\hline
\end{tabular}

Notes.

a XMM-Newton exposures are quoted for the EPIC-pn/each of the EPIC-MOS detectors.

b NUSTAR exposures are quoted for each of the focal plane modules.

Current results indicate the majority of AGNs may host rapidly rotating black holes, although the sample is still fairly small, and not yet well defined in a statistical sense.

However, the identification of these spectral features with relativistic disk reflection is not without controversy. In particular, scenarios fully dominated by absorption and reprocessing from material that is relatively distant to the AGN have frequently been proposed as alternative interpretations to relativistic disk reflection (e.g., Miller et al. 2008, 2009; Sim et al. 2010). If these absorbing and scattering structures are allowed sufficiently complex geometries, such models are also able to reproduce the observed X-ray spectra. Many AGNs do indeed display evidence of partially ionized absorption in X-rays (e.g., Blustin et al. 2005), as well as evidence of reflection from distant, dense material in the form of a narrow iron $\mathrm{K} \alpha$ emission line (e.g., Bianchi et al. 2009) and a number also display evidence for variable absorption (e.g., Risaliti et al. 2002). The contributions of this absorption can be difficult to fully disentangle from relativistic disk reflection in some cases, particularly without access to sensitive hard X-ray ( $>10 \mathrm{keV})$ coverage.

Since the launch of the Nuclear Spectroscopic Telescope Array (NuSTAR; Harrison et al. 2013), NGC 1365 has become central to the debate over the contribution of emission from the inner disk in AGNs. NGC $1365(z=0.0055, D \sim 20 \mathrm{Mpc})$ is a well studied Seyfert 1.9 galaxy, hosting a $\sim 2 \times 10^{6} M_{\odot}$ black hole (Schulz et al. 1999; Kaspi et al. 2005) which displays evidence for a relativistically broadened iron line (Risaliti et al. 2009; Walton et al. 2010; Brenneman et al. 2013) indicative of a rapidly rotating black hole. However, it is also known to display complex and variable absorption (Risaliti et al. 2005a, 2005b, 2009; Maiolino et al. 2010). The unprecedented high-energy data quality and the continuous $\sim 3-80 \mathrm{keV}$ bandpass provided by NuSTAR is ideal for the study of X-ray reflection (e.g., Miller et al. 2013; Tomsick et al. 2014; Marinucci et al. 2014a; Parker et al. 2014c). Early in the mission, NuSTAR observed NGC 1365, coordinated with XMM-Newton (Jansen et al. 2001) for soft $\mathrm{X}$-ray coverage, detecting both a relativistic iron line and a strong hard excess. The hard X-ray data from NuSTAR displayed excellent consistency with the prediction of the disk reflection interpretation from the XMM-Newton data and revealed that a Compton-thick absorber would be required to reproduce the high-energy data without invoking disk reflection. The presence of such material was found to be inconsistent with the levels of reprocessing observed, either from neutral or partially ionized material (Risaliti et al. 2013), providing a strong confirmation of the contribution from relativistic disk reflection. However, based on a set of simulated spectra, Miller \& Turner (2013) subsequently challenged this conclusion, suggesting that distant absorption/reprocessing could yet explain the spectra observed from NGC 1365 and claiming that AGN spins cannot be measured at all.

Here we present results from the full series of four coordinated observations of NGC 1365 performed by NuSTAR and XMMNewton, the first of which was initially presented in Risaliti et al. (2013). These observations probe an unprecedented range of absorption states with high signal-to-noise $(\mathrm{S} / \mathrm{N})$ data, and also reveal a persistent contribution from the inner accretion disk. We use these observations to disentangle the relative contribution of these processes and to determine the inner disk parameters. This work is structured as follows: the data reduction procedure is outlined in Section 2, our analysis is presented in Section 3, and the obtained results are discussed in section 4. Finally, we summarize our conclusions in Section 5.

\section{DATA REDUCTION}

NUSTAR and XMM-Newton have performed four coordinated observations of NGC 1365 to date, in 2012 July and December, and 2013 January and February, hereafter observations 1-4, respectively. Basic observation details are given in Table 1. Here we outline our data reduction procedure for these observations.

\subsection{NUSTAR}

The NUSTAR data were reduced using the standard pipeline, part of the NuSTAR Data Analysis Software v0.11.1 (NUSTARDAS; now part of the standard HEASOFT distribution), and instrumental responses from NuSTAR caldb v20130509 are used throughout this work. The unfiltered event files were cleaned with the standard depth correction, which significantly reduces the internal background at high energies, and South Atlantic Anomaly passages were excluded from our analysis. Source products were obtained from circular regions (radius $\sim 100^{\prime \prime}-120^{\prime \prime}$ ), and the background was estimated from a blank area of the same detector free of contaminating point sources. Spectra and light curves were extracted from the cleaned event files using XSELECT for both focal plane modules (FPMA and FPMB). Finally, the spectra were grouped such that each spectral bin contains at least 50 counts. The data from FPMA and FPMB are analyzed jointly in this work, but they are not combined together.

\subsection{XMM-Newton}

Data reduction was carried out with the XMM-Newton Science Analysis System (SAS v13.0.0) largely according to the standard prescription provided in the online guide. ${ }^{20}$ The observation data files were processed using EPCHAIN and EMCHAIN to produce calibrated event lists for the EPIC-pn (Strüder et al. 2001) and EPIC-MOS (Turner et al. 2001) detectors respectively. Source products were extracted from a circular region of $\sim 40^{\prime \prime}$ in radius, and the background was estimated from an area of the same CCD free of contaminating point sources. Light curves and spectra were generated with XMMSELECT, selecting only single and double events (single to quadruple events) for EPIC-pn (EPIC-MOS), excluding periods of high background flares (occurring predominantly at the end of the observations). The redistribution matrices and auxiliary response files were generated with RMFGEN and ARFGEN, while light curves were corrected for the background

\footnotetext{
20 http://xmm.esac.esa.int/
} 
count rate using EPICLCCORR. After performing the data reduction separately for each of the MOS CCDs and confirming their consistency, the spectra were combined using the FTOOL ADDASCASPEC. Finally, spectra were re-binned using the SAS task SPECGROUP to have a minimum $\mathrm{S} / \mathrm{N}$ of 5 in each energy bin.

\section{SPECTRAL ANALYSIS}

Throughout this work, spectral analysis is performed with XSPEC v12.8.0 (Arnaud 1996), and unless stated otherwise, parameter uncertainties are quoted at the $90 \%$ level of confidence for a single parameter of interest (i.e., $\Delta \chi^{2}=2.71$ ). The spectral agreement between XMM-Newton and NuSTAR is generally good across their common energy range ( $\sim 3-10 \mathrm{keV}$; e.g., Walton et al. 2013a, 2014; K. K. Madsen et al., in preparation), but we treat residual uncertainties in the flux calibration between the various detectors utilized by allowing variable constants to float between them, fixing the constant for the NuSTAR FPMA data to unity.

\subsection{Extreme Spectral Variability}

It is clear from a simple visual comparison of the average spectra obtained from the four observations, shown in Figure 1 (left panels), that our coordinated program caught a truly extreme level of spectral variability. Changes of over an order of magnitude can be seen at $\sim 2 \mathrm{keV}$, while at $\sim 10 \mathrm{keV}$ the variability is much more modest, at most a factor of $\sim 2$. The variability at lower energies $(\lesssim 1 \mathrm{keV})$ is also more modest, although this is not surprising, as traditionally below $\sim 3 \mathrm{keV}$ the spectrum observed from NGC 1365 is dominated by diffuse thermal emission, resolved with Chandra (Wang et al. 2009), which should not vary on the timescales probed. Variability of this nature and magnitude is most readily explained with drastic changes in the line-of-sight absorption, which have previously been observed from NGC 1365. This is confirmed by our detailed spectral analysis that is presented in Section 3.3 (see also Parker et al. $2014 b$ ). However, in past high $\mathrm{S} / \mathrm{N}$ observations, the column density has always remained substantial, above $\sim 10^{23} \mathrm{~cm}^{-2}$ (e.g., Risaliti et al. 2009; Maiolino et al. 2010; Walton et al. 2010; Brenneman et al. 2013), occasionally increasing to the extent of becoming Compton thick (Risaliti et al. 2005b). Here, we are witnessing variations in the opposite sense, observing the source in an almost fully uncovered state for the first time.

The first of these four observations, which is also the most absorbed, displays residuals to a simple absorbed power-law continuum indicating the presence of relativistic disk reflection, i.e., a relativistically broadened iron line at $\sim 6 \mathrm{keV}$, and a prominent Compton hump at $\sim 30 \mathrm{keV}$ (Risaliti et al. 2013). Here, we apply the same continuum to the average spectra from all four observations, consisting of a power law with $\Gamma=1.75$ modified by partially covering neutral absorption (using PCFABS), with both the column and the covering fraction free to vary between observations. This is fit to the 2.5-4, $7-10$, and $50-80 \mathrm{keV}$ energy ranges, in order to fit the AGNdominated continuum and to avoid the most prominent reflection features. Although formally only a visual aid, the residuals in the 3-80 keV energy range are extremely similar for each observation (see Figure 1, right panels), despite the massive changes in absorption column density inferred (ranging from $\sim 1-25 \times 10^{22} \mathrm{~cm}^{-2}$ ). Relativistically broadened iron emission lines, extending down to $\sim 4 \mathrm{keV}$, and strong Compton humps are observed in each case, which are clearly independent of the line-of-sight absorption. These residuals are not resolved by fitting this simple absorbed power-law model to the full 2.5-80 keV bandpass considered here.

In addition to the broad reflection residuals, two pairs of resolved narrow absorption lines at $\sim 7$ and $8 \mathrm{keV}$ from ionized iron can also be seen in all observations. These lines have been observed repeatedly from NGC 1365 (e.g., Risaliti et al. 2005a; Brenneman et al. 2013) and will be studied in detail in subsequent work (E. Nardini et al., in preparation). However, although these features are not the main focus of this work, we do point out that they primarily cause the sharp drop in the spectrum just below $\sim 7 \mathrm{keV}$, the depth of which is essentially independent of the neutral absorption column. This should not be confused with either the iron edge associated with neutral absorption, or the blue wing of the iron line. The blue wing of the line in fact continues to higher energies and can still be observed at $\sim 7.5 \mathrm{keV}$.

\subsection{Linking the Broad Iron Line and the Compton Hump}

Although we show the time-averaged spectra from each observation in Figure 1 for clarity, NGC 1365 is known to display both flux and spectral variability on relatively short timescales in soft X-rays ( tens of ks; e.g., Risaliti et al. 2009). As shown in Figure 2, such behavior is clearly displayed during our observations. Following Risaliti et al. (2013), we split each observation into four separate periods (resulting in 16 periods in total, as highlighted in Figure 2), which are determined by changes in intrinsic flux, as is roughly indicated by the $5-10 \mathrm{keV}$ count rate, and/or changes in spectral shape, as is roughly indicated by the hardness ratio between the 3-5 and 5-10 keV energy bands, and extracted spectra for each of these. For the first observation, the selected periods are the same as those used in Risaliti et al. (2013), for consistency. The data reduction follows that described in Section 2 for each period apart from the fourth period of observation 3 (P12 in Figure 2, the brightest part of the least absorbed observation). During this period, the observed XMM-Newton count rates were formally just in excess of the recommended limits for pile-up. Although comparison of the pattern distribution suggests any pile-up effects below $10 \mathrm{keV}$ are still minimal, to be conservative, we extract $X M M$ Newton spectra using an annular region for this period, with the same outer radius as the circular regions used otherwise, but with the innermost 6 " of the point spread function removed for $E P I C$-pn, and the innermost $10^{\prime \prime}$ removed for EPIC-MOS.

Following the same procedure as above for each of these individual periods results in the same residuals highlighted in Figure 1 in each case (albeit at lower $\mathrm{S} / \mathrm{N}$ ), even though the individual periods sample an even broader range of absorption states (see below). In our further analysis, we only consider the XMM-Newton data above $2.5 \mathrm{keV}$ in order to avoid the diffuse thermal contribution and to focus on these reflection features, and the NUSTAR data are modeled over the $4-79 \mathrm{keV}$ energy range. The only exception to this is the EPIC-MOS data from observation 3 , in which narrow residuals are observed just above $2.5 \mathrm{keV}$, most likely associated with calibration of the instrumental edges at slightly lower energies; these data are therefore modeled above $2.7 \mathrm{keV}$.

A key expectation of the relativistic reflection model is that the strength of the iron line and the high-energy reflection hump relative to the continuum are positively correlated. In order to test this prediction in a model-independent manner, we applied two purely phenomenological models to each of our 16 broadband spectra. The first consists of an absorbed 

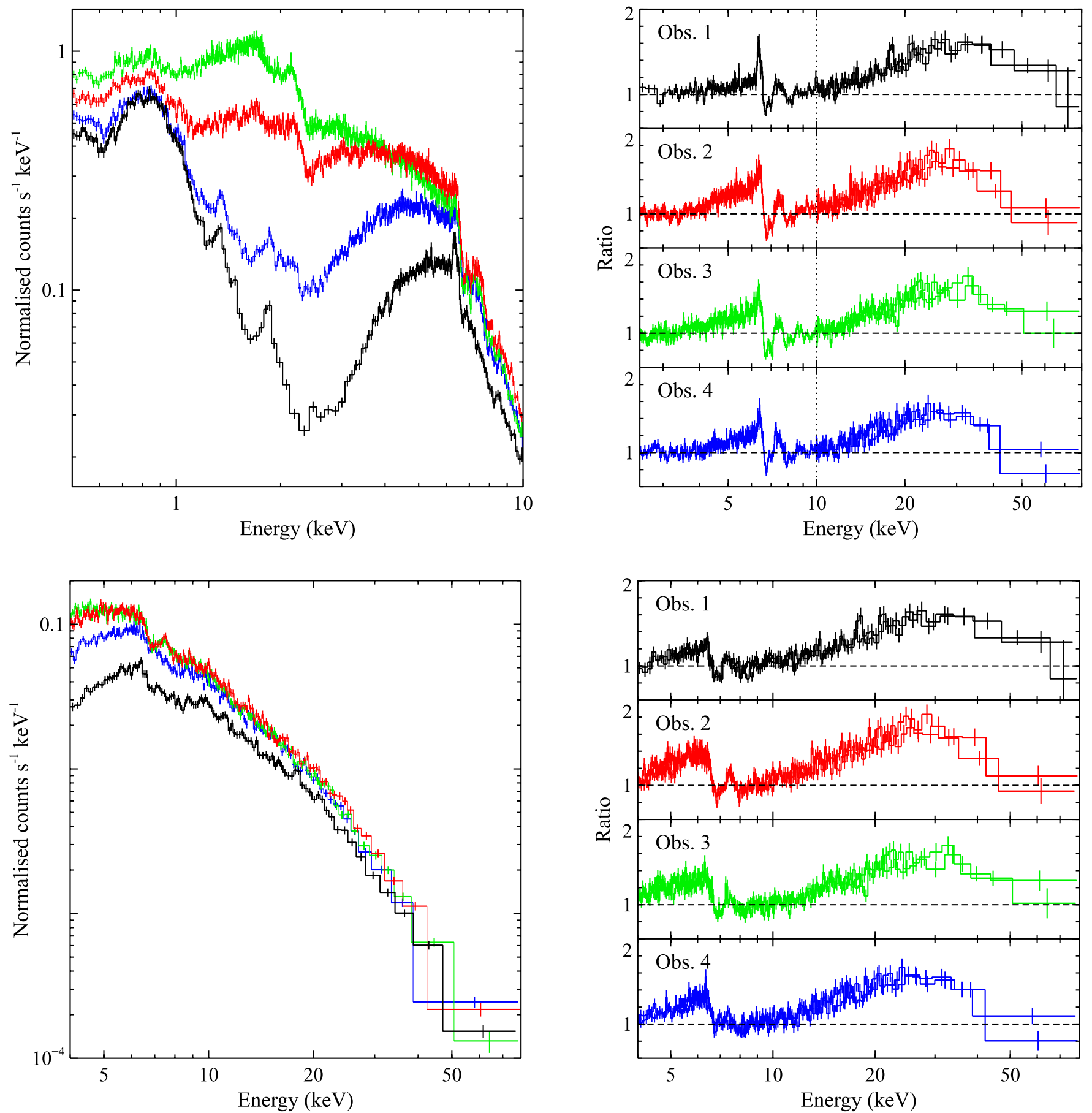

Figure 1. Top-left panel: time-averaged XMM-Newton EPIC-pn spectra from each of the four coordinated NuSTAR+XMM-Newton observations of NGC 1365, demonstrating the extreme spectral variability displayed. Observations 1,2,3, and 4 are shown in black, red, green, and blue, respectively. Top-right panel: residuals of a simple $\Gamma=1.75$ power-law continuum, modified by partially covering neutral absorption and applied to the $2.5-4,7-10$, and $50-80$ keV energy ranges. For clarity, we show the XMM-Newton EPIC-pn data below $10 \mathrm{keV}$ and the NuSTAR FPMA/FPMB data above $10 \mathrm{keV}$. The same hallmarks of reflection from the inner accretion disk, i.e., a relativistically broadened iron line at $\sim 6 \mathrm{keV}$ and a strong Compton hump at $\sim 30 \mathrm{keV}$ are seen in each of the four observations, despite the extreme variation in the line-of-sight absorbing column. Bottom panels: as for the top panels, but now displaying only the NuSTAR data, further highlighting the reduced variability at high energies compared to that seen at $\sim 2 \mathrm{keV}$, and the detection of the broad iron line in these data. The narrow component of the iron emission is less visually prominent in the NUSTAR data owing to the coarser spectral resolution in the iron K $\alpha$ bandpass in comparison to XMM-Newton. In the left panel, only the data from FPMA is shown for clarity. The data in all panels have been rebinned for visual purposes.

(A color version of this figure is available in the online journal.)

power-law continuum, a broad Gaussian emission line to treat the iron emission, and a narrower Gaussian absorption line to treat the strongest of the ionized iron absorption features and to avoid strong residuals which may alter the best-fit values of the other components, while the second consists simply of a power law and a neutral reflection continuum (PEXRAV; Magdziarz \& Zdziarski 1995). We simply use a single Gaussian line to treat the ionized iron $\mathrm{K} \alpha$ absorption here as, individually, the spectra from the 16 selected periods do not have sufficient $\mathrm{S} / \mathrm{N}$ to separate and to constrain all four of the absorption lines visible in 


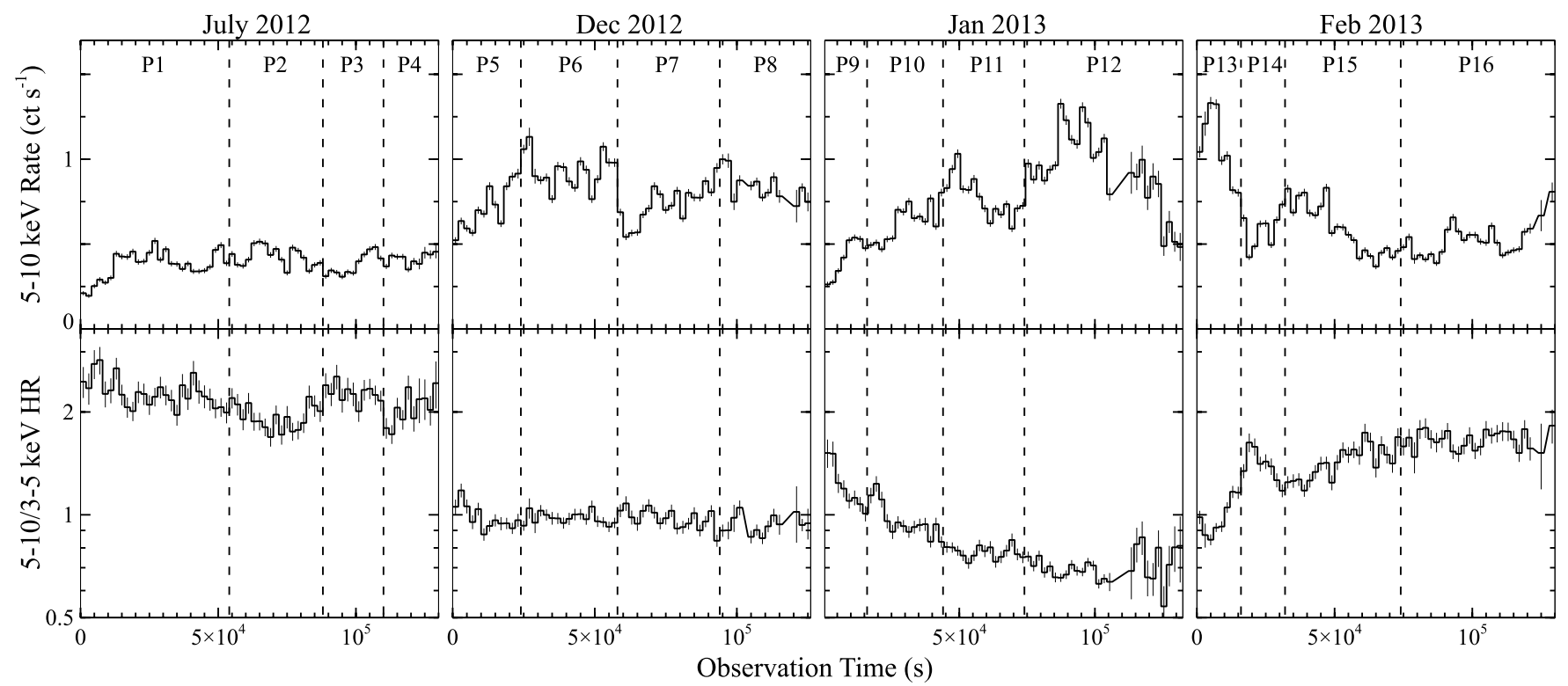

Figure 2. Top panels: the 5-10 keV XMM-Newton EPIC-pn light curves observed from NGC 1365 for each of the four observations. Bottom panels: the 5-10/3-5 keV hardness ratio light curves. The vertical dashed lines indicate the 16 periods selected for our time-resolved analysis (four periods are defined within each observation), based on either changes in the 5-10 keV light curve, and/or the 5-10/3-5 keV hardness ratio.

Figure 1 simultaneously, and the two weaker iron $\mathrm{K} \beta$ lines have a negligible effect on this analysis. The first model is applied to the data below $10 \mathrm{keV}$, and the second is applied to the data above $10 \mathrm{keV}$, with only the parameters of the intrinsic powerlaw continuum required to be the same in each energy range. In this way, the two interesting components are treated completely independently. For simplicity, we assume solar abundances for the reflection at this stage. The fits obtained with this simple procedure give a reduced $\chi^{2}<1.2$ in all cases, and do not show large residual features. We then estimated the equivalent widths of the broad Gaussian components, and the ratio $R$ between the reflection and intrinsic power-law normalizations.

The results are plotted in Figure 3. Although there is some scatter, likely related to degeneracies arising from the simplistic analysis, a significant positive correlation is still clear from the data, demonstrating a clear link between the strength of the Compton hump and the strength of the iron emission. The Kendall's $\tau$ correlation coefficient for these parameters is $\tau=0.55$, implying that the null hypothesis (no correlation) can be rejected at just over $3 \sigma$ confidence. We stress again that we have endeavored to treat the iron emission and the Compton hump independently here, and for both features, we consider quantities that assess their strength relative to the continuum, in order to ensure that our analysis would not artificially produce such a correlation. If we fit a power-law relation to the data, the best-fit index, $1.55 \pm 0.36$ is consistent with the relationship being linear, as would naturally be expected for two aspects of the same fundamental emission component. For illustration, we show the best-fit linear relation in Figure 3.

Finally, we confirm that the correlation observed between the broad iron emission and the Compton hump is not itself an artificial result of any parameter degeneracies related to this simple phenomenological analysis. Figure 4 shows the reflection parameter $R$ plotted against the column density, and the equivalent widths of the Gaussian included to account for the ionized absorption obtained with this analysis, which might influence the strength inferred for the broad iron emission. In contrast to the broad $\mathrm{Fe} \mathrm{K}$ equivalent widths, no visible correlations are readily apparent between either $R$ and $N_{\mathrm{H}}$ or

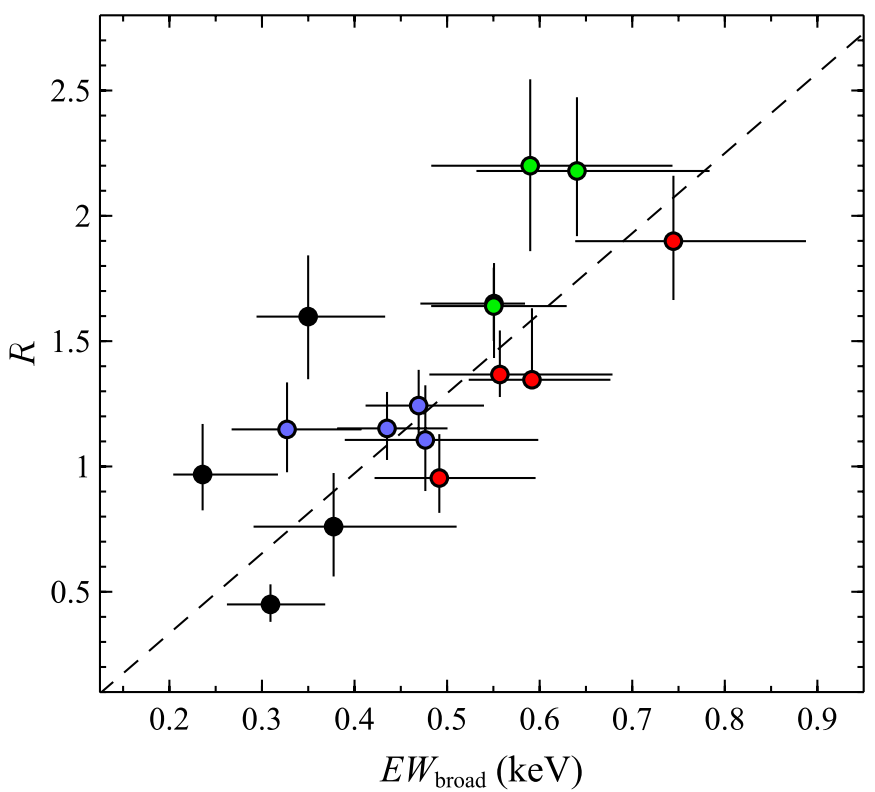

Figure 3. Correlation between the equivalent width $\left(\mathrm{EW}_{\text {broad }}\right)$ of the broad iron line, and the strength of the reflection continuum relative to the intrinsic power-law continuum, quantified as the reflection parameter $R$, inferred from the Compton hump for each of the 16 periods highlighted in Figure 2 using our simple phenomenological analysis. For clarity, the data from each observation has been color-coded to match Figure 1. The best-fit linear relation is shown with the dashed line.

(A color version of this figure is available in the online journal.)

$\mathrm{EW}_{\mathrm{abs}}$. Applying the Kendall correlation test again, we find the null hypothesis cannot be rejected at even the $95 \%$ level in either of these cases, confirming the intrinsic correlation between $R$ and $E W_{\text {broad }}$

\subsection{Relativistic Disk Reflection}

Having demonstrated the link between the iron line and the Compton hump using simple phenomenological models, we now undertake a detailed analysis of disk reflection utilizing a 

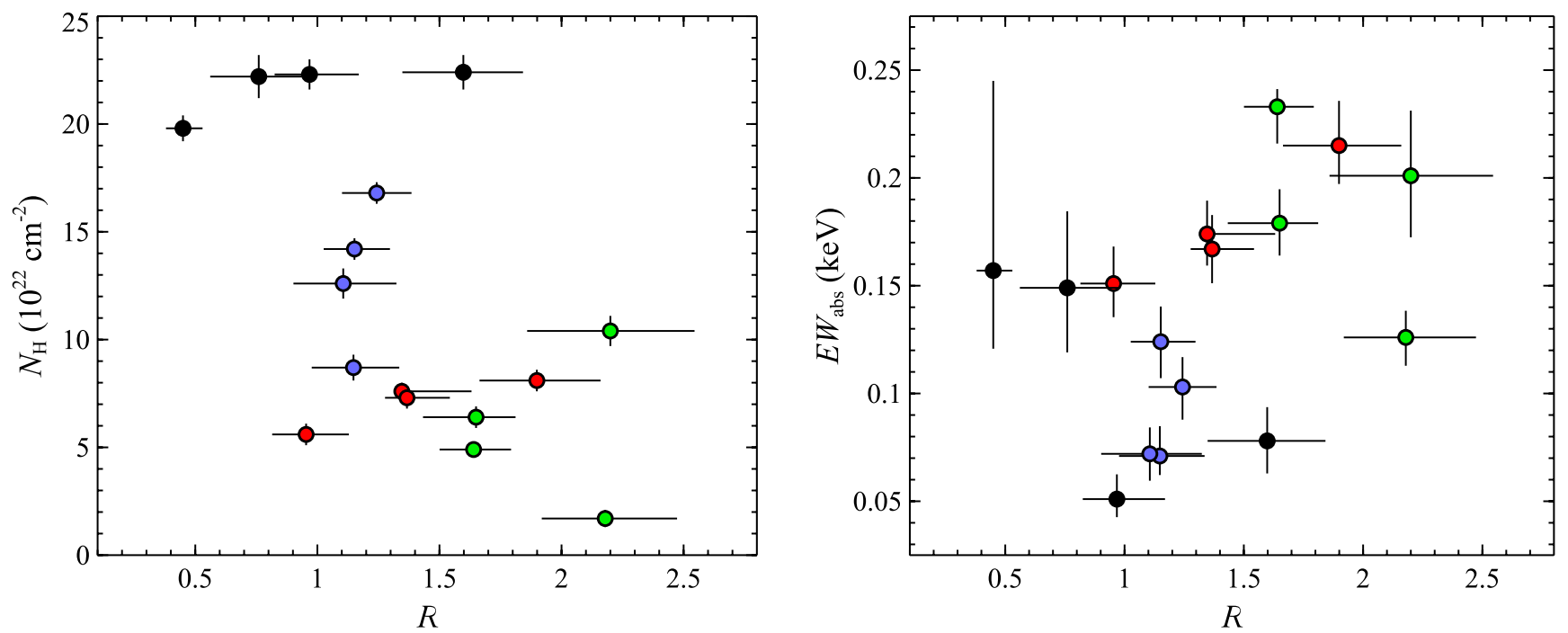

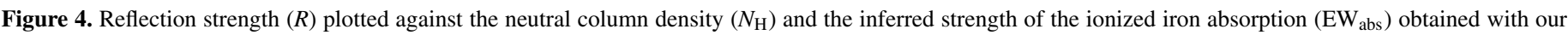

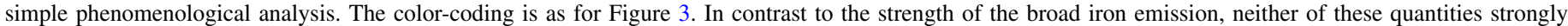

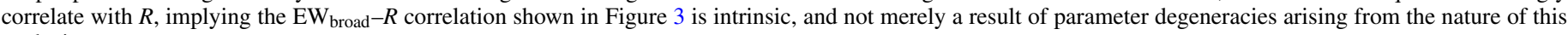
analysis.

(A color version of this figure is available in the online journal.)

detailed, physically self-consistent reflection model. We follow a similar approach to that undertaken in Risaliti et al. (2013), where we model each of the four periods identified from each observation simultaneously, in order to account for the spectral variability. However, our main aim in this work is to test the consistency of the results obtained with the relativistic disk reflection interpretation over the vastly differing absorption states, so we model each of the four observations independently.

\subsubsection{Model Setup}

In Risaliti et al. (2013), we made use of the REFLIONX reflection code (Ross \& Fabian 2005); however, in this work we make use of the XILLVER reflection code (García \& Kallman 2010) in order to test whether the results obtained previously depend on the exact reflection model used. Similar to REFLIONX, XILLVER self-consistently incorporates both the reflected continuum, including the high-energy $(\sim 30 \mathrm{keV})$ Compton reflection hump, and the accompanying line emission. The version of XILLVER used here also includes the subtle effects of viewing angle on the intrinsic reflected emission, and includes additional atomic physics. Besides the inclination $(i)$, the other key parameters are the iron abundance $\left(A_{\mathrm{Fe}}\right)$ and the ionization state (quantified as $\xi=4 \pi F / n$, where $F$ is the ionizing flux at the surface of the disk, and $n$ is the density) of the reflecting medium, and the photon index of the ionizing continuum (assumed to be a power-law). To fully model the relativistic disk reflection, the XILLVER model is convolved with the RELCONV kernel to account for the relativistic effects close to a black hole (Dauser et al. 2010). The key parameters for RELCONV are the spin of the black hole, $a^{*}$, the inclination of the accretion disk, and the index of the radial emissivity profile of the reflected emission, $q$, assumed here to have a power-law form. In order to measure the spin, we assume the accretion disk extends down to the ISCO; should this not be the case, any spin measurement obtained instead becomes a lower limit.

In addition to the disk reflection, we include an intrinsic power-law continuum. Both of these emission components are modified with a partially covering neutral absorber, and four multiplicative Gaussian absorption lines (using GABS) in order to account for the known ionized absorption visible in Figure 1 (with the higher $\mathrm{S} / \mathrm{N}$ in the combined data considered during this analysis, all four lines can now be independently constrained). We use simple Gaussian absorption lines in this work in order to also test whether the spin measurement depends strongly on our prior treatment of the ionized absorption with the XSTAR photoionization code (Kallman \& Bautista 2001). Finally, we also include a distant, neutral reflection component (again using XILLVER) in order to model the narrow component of the iron line, but this is not modified by either of the absorption components highlighted above, as this may well be reprocessed emission from the neutral absorber itself, which seems to be associated with the broad-line region (Risaliti et al. 2009), or could also plausibly arise from even more distant material, e.g., the narrow-line region and/or the dusty torus. For self-consistency, all inclination parameters, iron abundance parameters and photon indices are linked between the various components included in the model, and each of the four Gaussian absorption lines are required to have the same relative line broadening and outflow velocity.

As stated previously, for each observation we analyze the four periods identified in Figure 2 simultaneously. All parameters that are not expected to vary on observable timescales-i.e., the black hole spin, accretion disk inclination, the system iron abundance, and the normalization of the distant neutral reflection-are linked among the four periods, but they are allowed to vary overall. Following Risaliti et al. (2013), we also assume that the photon index remains constant. However, the neutral absorption column and the relative contribution of the intrinsic continuum and the disk reflection are allowed to vary between the periods. We also investigated allowing the covering fraction of the neutral absorber and the emissivity profile of the reflected emission to vary, but we found that doing so did not result in a significant statistical improvement for any of the observations. These parameters are therefore also linked among the periods, but they are allowed to vary overall. We also initially allowed the ionized absorption lines to vary, but found that this was only statistically required in the third observation (2013 January). In this case, we allow the line strengths to 

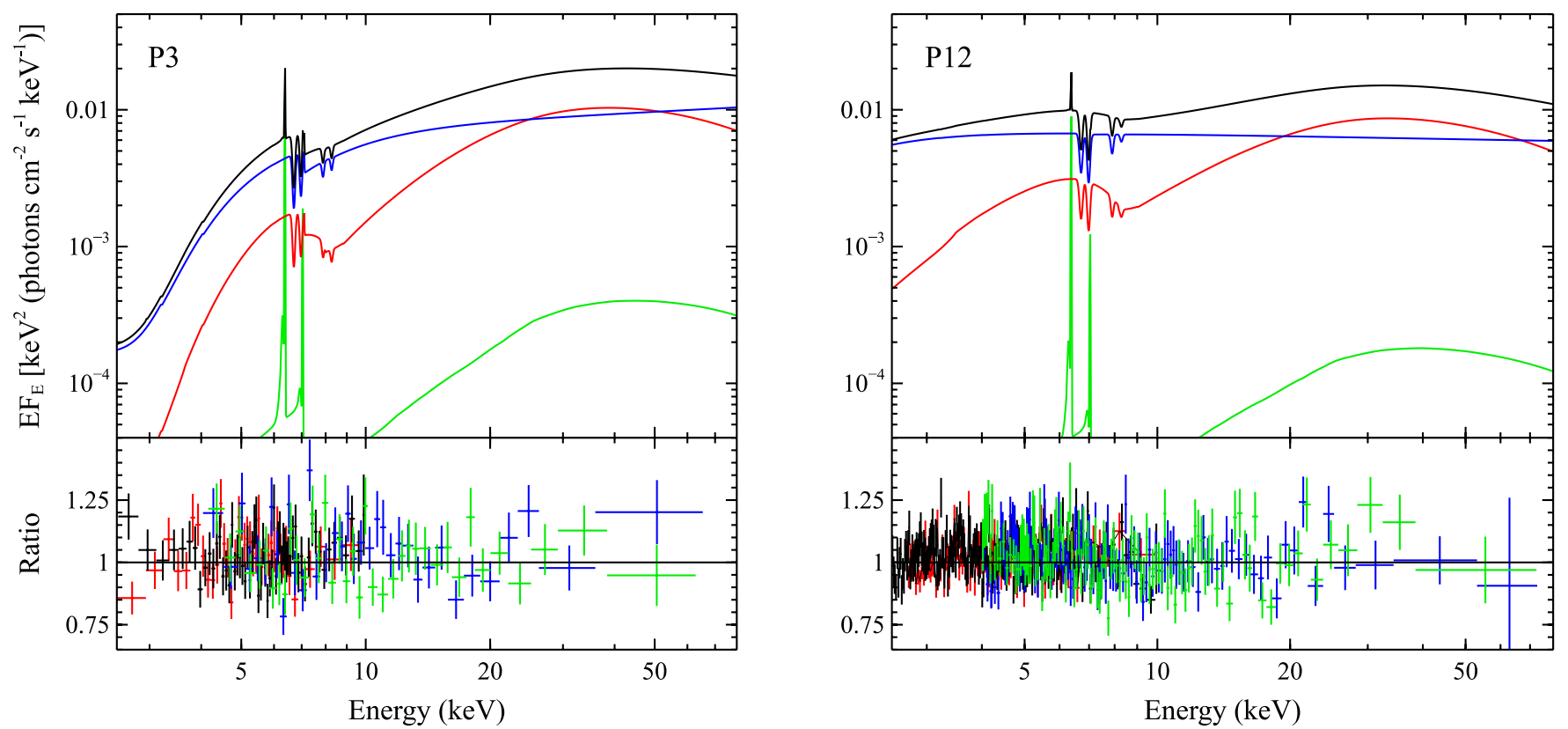

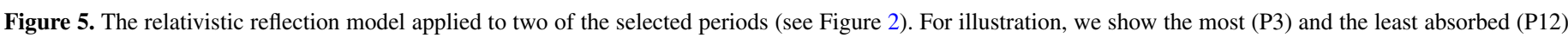

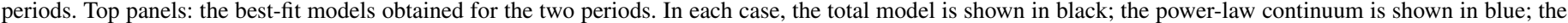

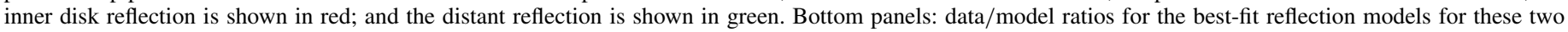
periods. The EPIC-pn, EPIC-MOS, FPMA, and FPMB data are shown in black, red, green, and blue, respectively.

(A color version of this figure is available in the online journal.)

vary between the selected periods, but we assume the relative strengths of the associated $\mathrm{K} \alpha$ and $\mathrm{K} \beta$ lines, the line broadening, and the outflow velocity do not vary with time, although they are allowed to vary overall. For the other observations, the line strengths, widths, and the outflow velocities are assumed to be constant with time, but again, they are free to vary overall.

Since the intrinsic flux changes are only moderate within each individual observation (see the 5-10 keV light curves in Figure 2), we assume the disk ionization does not vary between the selected periods, and we also limit the ionization to the range of $\log \xi_{\text {disk }}<3$. We do this to exclude unphysical reflection-dominated solutions at low spin, which require an extremely ionized accretion disk $\left(\log \xi_{\text {disk }} \geqslant 3.5\right)$ and a very hard intrinsic power-law continuum $(\Gamma \sim 1.5)$, only contributing significantly at the highest energies probed $(E \gtrsim 50 \mathrm{keV})$. Such scenarios can be rejected in the standard disk reflection paradigm, since the enhanced light bending required to produce reflection-dominated spectra in turn requires the corona to be located within a few gravitational radii $\left(R_{\mathrm{G}}\right)$ of the black hole, which is only plausible when the spin of the black hole is very high (Miniutti \& Fabian 2004). However, this is not yet self-consistently incorporated into the disk reflection models currently available, so such solutions must be excluded manually. For comparison, we note that such solutions had previously been allowed when computing the spin constraint presented in Risaliti et al. (2013). In any case, the ionization of the disk is only expected to be moderate, based on results obtained for other AGNs $\left(\log \xi_{\text {disk }}<3\right.$; Ballantyne et al. 2011; Walton et al. 2013b). As a final technical detail, we also assume that the crosscalibration constants between the various detectors utilized in this work do not vary with time, except during observation 3, owing to the unique data reduction procedure for period 12 (see Section 3.2). In this case, only the first three periods $(9,10$, and $11)$ are required to have the same cross-calibration constants.

\subsubsection{Results}

We systematically apply this modeling procedure to each of the four coordinated observations independently, i.e., although we require many of the parameters to be the same for each of the periods identified within individual observations (as detailed above, see Figure 2), no requirement for any parameter to be the same between observations is imposed. This allows us to test the consistency of the results obtained from each of the observations for key physical parameters, specifically the disk inclination and the black hole spin (which should not change on the timescales probed), and therefore to test the robustness of the relativistic disk reflection interpretation with a consistent modeling procedure. With this approach, we obtained good fits for each observation, $\chi^{2} / \mathrm{DoF}=3837 / 3747(=1.02)$, $6801 / 6569(=1.04), 6067 / 5783(=1.05)$ and $5645 / 5689$ $(=0.99)$ respectively, and no obvious systematic residuals remain. We show the fits to two of the selected periods in Figure 5, for illustration, and the key continuum parameters obtained are presented in Table 2 (with the exception of the black hole spin and disk inclination which, as the main focus of this work, are presented separately in Table 3).

In Figure 6 (left panel), we show the spin and inclination constraints obtained from each of the four observations. It is clear that in each case the spin is inferred to be very high $\left(a^{*}>0.97\right)$, and the inclination is also inferred to be fairly high $\left(i \sim 60^{\circ}\right)$. The quantitative constraints obtained are presented in Table 3. The quoted uncertainties represent only the statistical uncertainties obtained, and they do not incorporate any systematic uncertainties associated with the modeling procedure itself, or the models used. For example, although we have not allowed such solutions in order to enforce some level of physical self-consistency at low spin, some of the high-ionization, reflection-dominated solutions might be considered physically plausible at high spins, and permitting 

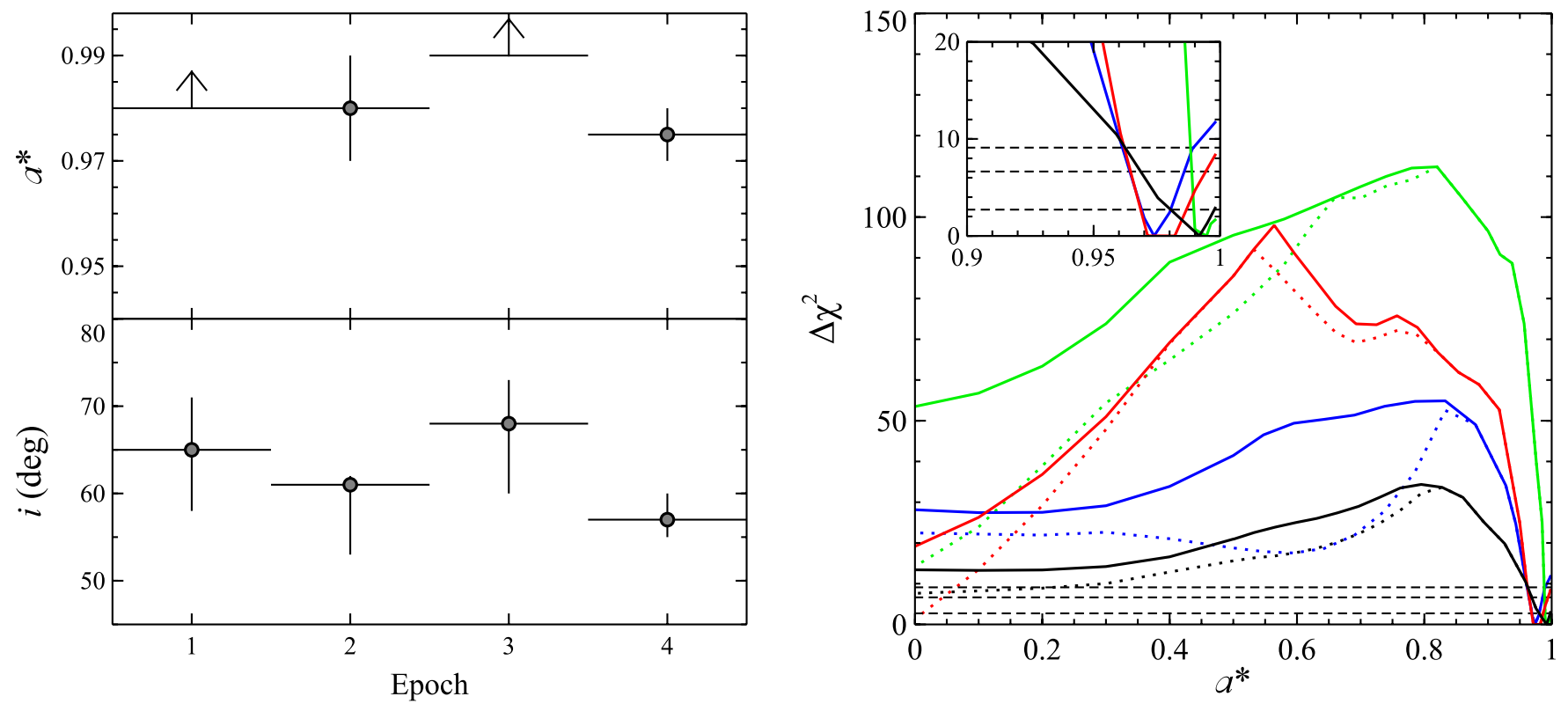

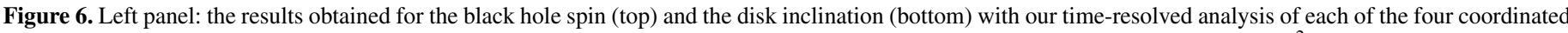

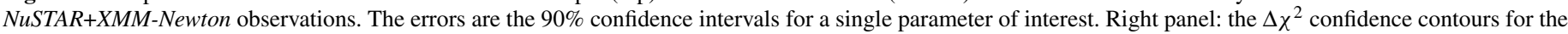

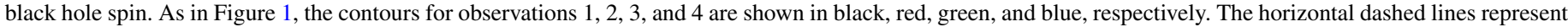

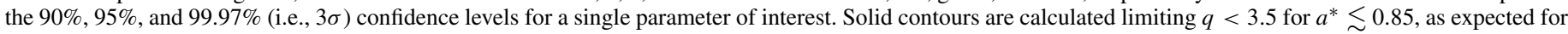
the standard disk reflection/light-bending model, while the dotted segments are calculated relaxing this constraint.

(A color version of this figure is available in the online journal.)

Table 2

Key Continuum Parameters Obtained for Each of the Four Observations of NGC 1365

\begin{tabular}{lcccc}
\hline \hline \multirow{2}{*}{ Parameter } & \multicolumn{4}{c}{ Observation } \\
\cline { 2 - 5 } & 1 & 2 & 3 & 4 \\
\hline$\Gamma$ & $1.84_{-0.04}^{+0.02}$ & $1.99_{-0.20}^{+0.04}$ & $2.05_{-0.03}^{+0.05}$ & $2.07_{-0.05}^{+0.04}$ \\
$N_{\mathrm{H}}(1)^{\mathrm{a}}$ & $25.2 \pm 0.7$ & $7.7_{-0.8}^{+0.5}$ & $12.5_{-1.3}^{+1.2}$ & $7.7_{-0.4}^{+0.3}$ \\
$N_{\mathrm{H}}(2)^{\mathrm{a}}$ & $21.8 \pm 0.6$ & $7.4_{-0.8}^{+0.5}$ & $6.3_{-0.8}^{+1.2}$ & $12.7_{-0.6}^{+0.8}$ \\
$N_{\mathrm{H}}(3)^{\mathrm{a}}$ & $26.4 \pm 0.9$ & $7.1 \pm 0.5$ & $2.9 \pm 0.4$ & $14.0 \pm 0.5$ \\
$N_{\mathrm{H}}(4)^{\mathrm{a}}$ & $22.5 \pm 0.9$ & $6.3_{-0.8}^{+0.4}$ & $1.3_{-0.2}^{+0.3}$ & $18.3 \pm 0.6$ \\
$C_{\mathrm{f}}(\%)$ & $97.2 \pm 0.2$ & $88_{-2}^{+1}$ & $80_{-4}^{+3}$ & $95.5_{-0.4}^{+0.3}$ \\
$\log \xi_{\text {disk }}$ & $1.6_{-1.5}^{+0.2}$ & $>1.5$ & $1.7_{-1.2}^{+0.1}$ & $1.7_{-1.1}^{+0.1}$ \\
$q$ & $7.2_{-1.8}^{+0.5}$ & $6.5_{-0.5}^{+0.2}$ & $8.3_{-2.1}^{+0.2}$ & $5.0_{-0.4}^{+0.5}$ \\
$A_{\mathrm{Fe}} /$ solar & $5.0_{-0.7}^{+2.3}$ & $5.0_{-0.8}^{+1.4}$ & $5.0_{-0.2}^{+1.0}$ & $3.8_{-0.3}^{+0.4}$ \\
\hline$\chi^{2} /$ dof & $3837 / 3747$ & $6801 / 6569$ & $6067 / 5783$ & $5645 / 5689$ \\
& $(=1.02)$ & $(=1.04)$ & $(=1.05)$ & $(=0.99)$ \\
\hline
\end{tabular}

Notes.

${ }^{\text {a }}$ Column densities 1-4 refer to the four periods selected within each respective observation (e.g., $N_{\mathrm{H}}$ (3) for observation 4 refers to P15 in Figure 2), and they are quoted in units of $10^{22}$ atom $\mathrm{cm}^{-2}$.

$\mathrm{b}$ The ionization parameter is quoted in units of $\mathrm{erg} \mathrm{cm} \mathrm{s}^{-1}$.

these solutions could broaden the statistically allowed parameter range to some extent. However, we stress that, based on the physical considerations discussed above, this would not change the ultimate requirement for high spin. Furthermore, we note that when the spin constraints have been investigated for a variety of input model assumptions for other accreting black holes (both binaries and AGNs) in which high spin has been inferred, the overall requirement for high spin has generally been found to be robust (e.g., AGNs: Brenneman et al. 2011; galactic binaries: Reis et al. 2012b; Walton et al. 2012; Tomsick et al. 2014).
Table 3

The Black Hole Spin and Disk Inclination Constraints Obtained for Each of the Four Observations of NGC 1365

\begin{tabular}{lcc}
\hline \hline Observation & $a^{*}$ & $\begin{array}{c}i \\
\left({ }^{\circ}\right)\end{array}$ \\
\hline 1 & $>0.98$ & $65_{-7}^{+6}$ \\
2 & $0.98 \pm 0.01$ & $61_{-8}^{+1}$ \\
3 & $>0.99$ & $68_{-8}^{+5}$ \\
4 & $0.975 \pm 0.005$ & $57_{-2}^{+3}$ \\
\hline
\end{tabular}

In addition, although both reflection models utilized here and in Risaliti et al. (2013) compute reflected spectra from simple, constant-density slabs (Ross \& Fabian 2005; García \& Kallman 2010), models produced with more complex density profiles which might be expected for real accretion disks-e.g., models computed from slabs of material in hydrostatic equilibrium (Nayakshin et al. 2000)—also produce broadly similar results, i.e., strong iron emission that is narrow in the restframe and a prominent Compton hump. Thus, there is no reason to expect that such models would produce noticeably different results from those obtained here, as the line broadening will still naturally be dominated by the inner extent of the disk. Indeed, Ballantyne et al. (2001, 2004) demonstrate the qualitative similarity of models assuming a constant density and those invoking more complex density profiles across the bandpass incorporating the iron $\mathrm{K} \alpha$ emission and the Compton reflection hump, further supporting our assertion that the key results presented here regarding the black hole spin and disk inclination are robust to these particular details. Instead, we expect that the iron abundance might be the parameter most sensitive to the assumptions regarding the density profile as, for certain illumination scenarios, models with different density treatments can make different predictions for the strength of the iron emission relative to the continuum (Nayakshin et al. 2000). 
The quoted uncertainties also do not incorporate systematic uncertainties related to the key assumptions inherent to the measurement of black hole spin, for example (and most importantly) that the accretion disk truncates at the ISCO (or more precisely that there is negligible reflected emission from within the ISCO). However, simulations suggest that this is generally a fairly good assumption, particularly at high spin (Reynolds \& Fabian 2008). Nevertheless, even with these formal statistical uncertainties, both the spin and the inclination obtained independently from each observation all agree at the $3 \sigma$ level of confidence or better, clearly demonstrating that the reflection interpretation naturally gives consistent results for the key physical quantities, despite the vast range of absorption states that are observed. A full assessment of the model/systematic uncertainties when self-consistently treating all the data will be presented in future work (L. W. Brenneman et al., in preparation). In addition to the key geometric parameters, the iron abundance should also remain constant on observable timescales, and again the values obtained display a good level of consistency, agreeing at the $3 \sigma$ level or better.

We also show the $\Delta \chi^{2}$ confidence contours for the spin parameter obtained from each of the observations in Figure 6 (right panel) in order to demonstrate that the best-fit solutions obtained are always consistent with being the global minimum when considering prograde spin, ${ }^{21}$ despite the moderately complex $\chi^{2}$ landscape. Similar to recent analyses by Steiner et al. (2012) and King et al. (2014), when computing these contours, we restrict the emissivity index to $q<3.5$ for $a^{*} \lesssim 0.85$, as solutions with steep emissivities are only physically plausible for rapidly rotating black holes in the disk reflection/light-bending paradigm (e.g., Wilkins \& Fabian 2012; Dauser et al. 2013). Although something of an arbitrary value, we make this transition at $a^{*} \sim 0.85$ for two reasons: first, because this is always a low enough spin that it does not influence the statistical constraint obtained around the best-fit solution; and second, to avoid large discontinuities in the plotted contours, since the best-fit models for each of the four observations all give $q \lesssim 3.5$ at $a^{*} \sim 0.85$. While each individual case is slightly different, in general, the $\chi^{2}$ contours obtained from each observation with the above model setup favor a high spin, with a rapid initial rise in $\chi^{2}$ followed by a slow decline as lower spins are considered.

Finally, we also demonstrate the effect of relaxing the constraint on the emissivity index for low spins (dotted contour segments). This generally results in slightly better fits in this regime, although the high-spin solution is still statistically favored for most of the observations. In the case of observation 2, however, the decrease in $\chi^{2}$ toward low spin becomes such that the best fit at $a^{*}=0$ is formally statistically comparable to the best-fit high-spin solution, owing to strong degeneracies among the various model parameters (when they are allowed sufficient freedom). Interestingly, this is the observation displaying the least spectral variability, which may in fact serve to exacerbate parameter degeneracies for the complex model considered here. Evidence for this can also be seen in Table 2, where several key continuum parameters are not as well constrained as for the other observations. However, in addition to requiring a steep $(q>4)$ emissivity index, this low spin solution is also reflection-dominated, despite our best efforts to exclude such

\footnotetext{
21 We do not consider retrograde spin in this work, as to date there is no evidence for an active galaxy with such a spin orientation (Walton et al. 2013b). Although Cowperthwaite \& Reynolds (2012) initially suggested that 3C 120 might have a retrograde spin, Lohfink et al. (2013) later demonstrated this is unlikely to be the case.
}

solutions in this regime. We therefore discard this low-spin solution on physical grounds. Models that self-consistently incorporate spin-dependent effects for the emissivity and the reflected contribution (e.g., M. Dovciak et al., in preparation) will be utilized in the future.

\section{DISCUSSION}

We have presented a spectral analysis of the full set of four coordinated NUSTAR and XMM-Newton observations of the well known Seyfert galaxy NGC 1365, focusing primarily on the 2.5-79 keV energy range in which the nuclear emission dominates. Although NGC 1365 has typically been observed to be obscured by a fairly substantial column (e.g., Risaliti et al. 2005a; Walton et al. 2010; Brenneman et al. 2013), as shown in Figure 1, these four high $\mathrm{S} / \mathrm{N}$ observations caught the source in an unprecedented range of absorption states, including states with little line-of-sight absorption to the central nucleus $\left(N_{\mathrm{H}}<10^{22.5} \mathrm{~cm}^{-2}\right)$. The full implications for the structure of the absorption will be explored in future work (E. Rivers et al. 2014, in preparation). Despite this range of absorption, the observed spectrum from each observation displays the same hallmarks of reflection from the innermost regions of the accretion flow, i.e., relativistically broadened iron emission and a strong Compton hump. Furthermore, we also find that the breadth of the line is independent of the level of absorption observed. The simplest interpretation is that these features are not directly associated with line-of-sight absorption, providing yet more compelling evidence for the contribution of relativistic disk reflection.

Through time-resolved spectroscopy, similar to recent analyses of other bright AGNs (e.g., Reis et al. 2012a; Parker et al. 2014a; Marinucci et al. 2014b), we have demonstrated a clear link between the broad iron emission and the Compton hump (Figure 3), as expected if these features are two aspects of the same underlying component, as in the relativistic reflection model. We also examined the consistency of the results obtained for key physical parameters through self-consistent modeling of this reflection, allowing for variable, partially covering absorption. In spite of the complexity of the behavior displayed by NGC 1365, consistent results are obtained for both the disk inclination and the black hole spin in each case, as shown in both Figure 6 and Table 3. The statistical agreement between the results obtained is good even before considering the potential systematic uncertainties associated with these measurements. This is an important consistency check for the disk reflection interpretation, since these physical parameters should not vary on observable timescales. The fact that similar results are naturally obtained from our reflection analysis provides further confidence in the results obtained, and thus in the overall reflection paradigm, our ability to view the innermost regions of the accretion flow and to measure black hole spin for active galaxies.

\subsection{Distant Reprocessing}

In response to the results presented by Risaliti et al. (2013), Miller \& Turner (2013) performed a series of simulations arguing that a combination of partially covering absorption and distant reprocessing could produce spectra similar to those observed without any contribution from relativistic disk reflection. A similar model had been investigated by Risaliti et al. (2013), and was found to require three independent absorption components, one of which was Compton-thick, in addition to Compton 
reflection from distant material in order to reproduce both the $\sim 4-8 \mathrm{keV}$ spectral complexity (otherwise interpreted as relativistically broadened iron emission) and the strong hard-excess at $\sim 20 \mathrm{keV}$. This model was able to adequately reproduce the data from the first observation in a statistical sense, and we confirm that this formally remains the case for the additional observations by repeating the time-resolved analysis undertaken in Risaliti et al. (2013) for each, constructing models in which the relativistic disk reflection contribution is replaced by additional partially covering absorption components.

Consistent with Risaliti et al. (2013), we find that two additional absorption components (making three in total) are generally required to fit the data $\left(\chi^{2} /\right.$ dof $=3853 / 3721$, $6940 / 6544,6093 / 5763$, and 5664/5664 for the four observations, respectively), in addition to a distant Compton reflection component to account for the narrow iron line (modeled again with XILLVER). These fits are still poorer than for the relativistic reflection model (see Table 2). Removal of one of these absorption components degrades the fit by $\Delta \chi^{2}=30-70$ (for eight fewer free parameters) in each of the observations, with the third absorber being least statistically significant in the least absorbed observation (Obs. 3, although an F-test still suggests it is significant at greater than the $99.9 \%$ statistical level). The column densities and covering fractions for the two additional absorbing components are found to range from $N_{\mathrm{H}, 1}=3.5-5 \times 10^{24} \mathrm{~cm}^{-2}$ and $C_{\mathrm{f}, 1}=0.5-0.7$ (Compton-thick), and $N_{\mathrm{H}, 2}=1-5 \times 10^{23} \mathrm{~cm}^{-2}$ and $C_{\mathrm{f}, 2}=0.4-0.8$, respectively, while the third component displays very similar values to those obtained with the relativistic reflection model (Table 2), again displaying more than an order of magnitude of variation in column density. Regardless of whether two or three absorption components are used, one is strongly required to be Comptonthick and relatively constant, and one is strongly required to be highly variable. However, this model was ultimately rejected on physical grounds in Risaliti et al. (2013). We summarize the main points below, and we refer the reader to that work for a more detailed discussion.

In the absorption-dominated scenario, the intrinsic luminosities inferred are significantly higher than the observed ones, due to the effects of Compton scattering. The exact correction factor depends primarily on the geometry and global covering fraction $\left(C_{\mathrm{g}}\right.$; as seen from the X-ray source) of the dominant Compton thick absorber and may vary from a factor of $\sim 4-6$ for an almost totally covering absorber (i.e., large $C_{\mathrm{g}}$, in good agreement with Miller \& Turner 2013 despite the modeling differences), to $\sim 20-30$ for a geometrically thin gas ring (small $C_{\mathrm{g}}$ ). In all scenarios, the intrinsic luminosities are either inconsistent with those estimated from other indicators (smaller $C_{\mathrm{g}}$; in the thin-ring case, the total X-ray luminosity alone is close to the Eddington limit, and more than 1 order of magnitude higher than the luminosity inferred from the [O III] emission) or the observed level of reprocessed radiation (e.g., the narrow iron $\mathrm{K} \alpha$ flux, total radiation reprocessed in the infrared; larger $C_{\mathrm{g}}$ ).

Miller \& Turner (2013) argue in the higher $C_{\mathrm{g}}$ case that the intrinsic X-ray flux in the absorption scenario may be roughly similar to that inferred in the disk reflection scenario, given that the light bending required to explain the enhanced reflection and steep emissivity profiles likely mean that the fraction of the intrinsic X-ray flux lost over the event horizon is enhanced. However, the key point is that in the absorption scenario this flux does escape from the black hole and must be reprocessed. Furthermore, though Miller \& Turner (2013) suggest almost all AGNs (including type-1 sources) are affected by Compton-thick
Table 4

Relative and Absolute Line Fluxes for the Narrow Iron Emission for Each of the Four Observations of NGC 1365

\begin{tabular}{|c|c|c|c|c|}
\hline & \multicolumn{4}{|c|}{ Observation } \\
\hline & 1 & 2 & 3 & 4 \\
\hline $\mathrm{EW}_{\text {narrow }}(\mathrm{eV})$ & $57_{-11}^{+12}$ & $31 \pm 7$ & $31 \pm 8$ & $34_{-7}^{+8}$ \\
\hline$N_{\text {narrow }}{ }^{\mathrm{a}}$ & $1.1_{-0.2}^{+0.1}$ & $1.2_{-0.2}^{+0.3}$ & $1.1_{-0.2}^{+0.3}$ & $1.0 \pm 0.2$ \\
\hline
\end{tabular}

line-of-sight obscuration in X-rays, with covering factors close to unity (see also Tatum et al. 2013) and highlight that NGC 1365 does not display extreme levels of reprocessing relative to other AGNs, such comparison does not address the original point from Risaliti et al. (2013) that the observed level of reprocessing does not appear to match that expected from the absorption scenario in an absolute sense, not a relative sense. Therefore, the basic conclusion presented in Risaliti et al. (2013) remains valid.

\subsection{Viewing the Inner Disk}

In addition to these considerations, the observed spectral variability from the full data set also allows us to confirm the contribution of relativistic reflection from the inner disk through other physical arguments. Were the feature interpreted as the iron line to be produced primarily by partially covering line-of-sight absorption, rather than by disk reflection, there is no reason to expect a priori that such consistency in the inner disk parameters would be observed. Indeed, given the strong evolution clearly observed in the line-of-sight absorption, this would certainly not be expected, particularly for the black hole spin which is primarily constrained by the red-wing of the line, where the observed absorption variability has the largest effect (See Figure 1).

Furthermore, as is also visually apparent from Figure 1, the strength of the narrow iron emission, which likely does arise through distant reprocessing, varies relative to that of the broad iron emission. To quantify this, we construct a simple phenomenological model, replacing the distant and disk reflection components included in the self-consistent model used above (section 3.3) with a Gaussian emission line (with an intrinsic width of $10 \mathrm{eV}$ ) and a combination of a reflected continuum (PEXRAV) and a relativistic emission line (RELLINE), and we apply this model to the time-averaged broadband spectrum from each of the observations. The equivalent widths of the narrow emission line are presented in Table 4, confirming the variation in its strength relative to the broad line. This strongly suggests that these features are not different aspects of the same underlying emission component, particularly given that the absolute flux of the narrow line is consistent with remaining constant over the observed timescales (Table 4), as expected for distant emission.

Finally, we note that the detection of a short-timescale reverberation lag associated with the broad emission line further confirms this as emission from the inner regions of the accretion flow (Kara et al. 2014). Observation of similar short-timescale reverberation from features potentially associated with reflection from the inner disk has recently been found to be fairly common, both from the soft excess (Fabian et al. 2009; Emmanoulopoulos et al. 2011; De Marco et al. 2013; Fabian et al. 2013; Cackett et al. 2013) and the broad iron line (Zoghbi et al. 
2012, 2013; Kara et al. 2013), also strongly supporting an origin in the inner disk. Although reverberation from distant reprocessing material was again initially proposed as an alternative interpretation for the early AGN lag results (e.g., Miller et al. 2010; Legg et al. 2012), this no longer seems able to explain the complex lag phenomenology observed. In particular, high-frequency reverberation lags have now been detected from the broad iron $\mathrm{K} \alpha$ emission (e.g., Zoghbi et al. 2012; Kara et al. 2013), while similar iron $\mathrm{K} \alpha$ features are not seen in the low-frequency lags relevant to the distant reverberation interpretation (e.g., Kara et al. 2013), and the association of these low-frequency "hard" lags with the power-law continuum, rather than distant reverberation, has now been demonstrated (Walton et al. 2013c), a conclusion that is supported by comparison with galactic binaries (Kotov et al. 2001).

Indeed, the similarity of the broad iron features observed from both AGNs and Galactic BHBs, which are generally not heavily absorbed, in itself provides a compelling case for a disk reflection origin (Walton et al. 2012), and features consistent with relativistic disk reflection are also observed in AGNs with no evidence for obscuration (e.g., Nardini et al. 2011; Lohfink et al. 2012; Walton et al. 2013b). Furthermore, we have now detected relativistically broadened iron emission in a gravitationally lensed quasar (Reis et al. 2014), where microlensing studies independently constrain the size of the X-ray emitting region to be $R_{\mathrm{X}} \lesssim 10 R_{\mathrm{G}}$ (Dai et al. 2010), firmly ruling out the possibility of the broadened iron emission being produced by large, distant reprocessing structures. There is, therefore, now a substantial body of independent evidence suggesting that we are indeed viewing the inner disk in a significant population of AGNs. Our results firmly place NGC 1365 among this number (with the obvious exception of the Compton-thick phases occasionally displayed; Risaliti et al. 2005b).

\subsection{Black Hole Spin}

These results present a consistent picture, that even though NGC 1365 does clearly exhibit variable line-of-sight absorption, it also hosts a rapidly rotating black hole, viewed at moderately high inclination. Our analysis constrains the spin of NGC 1365 to be $a^{*}>0.97$ (based on the $90 \%$ statistical uncertainties). The quantitative results obtained for the spin here (Table 3) are consistent with the initial constraint presented in Risaliti et al. (2013) based on the first observation alone, despite subtle differences in our analysis, e.g., using a different reflection model and a different treatment for the ionized iron absorption, suggesting that the overall conclusion is not sensitive to these details. The spin constraints obtained here are also similar to the constraint obtained by Brenneman et al. (2013), and that implied from the inner radius obtained by Walton et al. (2010) through study of a single Suzaku observation, although in the former the inclination was not allowed to vary (we note that the assumed value was similar to those obtained here), and the latter simply assumed fully covering neutral absorption. Nevertheless, our results attest positively to the broad reliability of single-epoch spin measurements from reflection for active galaxies.

This is an important point, as it is difficult to collect the wealth of high-quality data across various epochs that can be available for Galactic binaries (e.g., Miller et al. 2012) for active galaxies, owing to both their longer variability timescales and their typically lower fluxes. In many cases, studies of AGN spin are therefore limited to single-epoch reflection measurements (e.g., Walton et al. 2013b). Indeed, in order for AGN spin measurements to progress toward ultimately providing strong tests of models for galaxy formation and/or SMBH growth (e.g., Berti \& Volonteri 2008; Dubois et al. 2013), substantially larger and better statistically defined samples of AGN spin measurements than are currently available (Walton et al. 2013b; Reynolds 2013) will be required, covering a broader range of redshift space, and hence pragmatically the reliance on such single-epoch reflection measurements will inevitably increase. ${ }^{22}$ The results presented here should be a cause for optimism that such progress can indeed be made with continued advances in X-ray instrumentation, e.g., Astro-H (Takahashi et al. 2012) and ATHENA+ (Nandra et al. 2013; Dovciak et al. 2013), and large observing programs, particularly if complemented by sensitive hard X-ray coverage similar to that provided by $N U S T A R$.

\section{CONCLUSIONS}

We present the first results from the full joint observing campaign undertaken by $N U S T A R$ and XMM-Newton on the well known Seyfert galaxy NGC 1365. The four coordinated observations reveal an extreme level of spectral variability, which primarily appears to be due to variable line-of-sight absorption. However, while changes in absorption have been observed in NGC 1365 before (e.g., Risaliti et al. 2005b; Connolly et al. 2014), our observations display relatively unobscured states with high $\mathrm{S} / \mathrm{N}$ for the first time. Despite the diverse range of absorption states displayed, each of the observations displays the same hallmarks of relativistic reflection from the inner accretion disk: a relativistically broadened iron line and a strong Compton reflection hump. These features therefore cannot be associated with line-of-sight absorption. Indeed, with a simple phenomenological analysis, we find that the strength of the relativistic iron line and the Compton hump relative to the intrinsic continuum are well correlated, despite treating each of these features independently, as expected if they are two manifestations of the same broadband reflection spectrum.

Following this simple analysis, we perform time-resolved spectroscopy with physically self-consistent disk reflection models, allowing for variable, partially covering absorption, in order to constrain the inner disk parameters. We treat each of the four observations independently in order to test the consistency of the results obtained for the key physical parameters, i.e., the black hole spin and disk inclination, which should not vary on observable timescales. Excellent consistency between these results obtained for these parameters from each of the four observations is found. Despite the recent controversial claim by Miller \& Turner (2013) that AGN spin cannot be measured, our results further demonstrate that it is possible to measure the spin of the black holes powering active galaxies, and we find that the central black hole in NGC 1365 is indeed rapidly rotating.

The authors would like to thank the referee for providing useful feedback, which helped improve the manuscript. This research has made use of data obtained with the NuSTAR mission, a project led by the California Institute of Technology (Caltech), managed by the Jet Propulsion Laboratory (JPL) and funded by NASA, and XMM-Newton, an ESA science

\footnotetext{
22 We note that in rare cases in the local universe it may still be possible to directly observe the thermal emission from the inner disk, from which it may be possible to constrain the spin through estimation of the emitting area, provided the black hole mass, disk inclination, and the relevant atmospheric corrections are known (Zhang et al. 1997; Done et al. 2013). However, given the disk temperatures involved for even the lowest mass AGN, even the small likelihood of being able to utilize this method decreases quickly with increasing redshift.
} 
mission with instruments and contributions directly funded by ESA Member States and NASA. We thank both the XMMNewton and the NUSTAR Operations, Software, and Calibration teams for support with the execution and analysis of these coordinated observations. This research was supported under NASA grant No. NNG08FD60C and has made use of the NUSTAR Data Analysis Software (NUSTARDAS) jointly developed by the ASI Science Data Center (ASDC, Italy) and Caltech (USA). P.A. acknowledges financial support from Conicyt ACT 1101, and P.G. acknowledges support from STFC (grant reference ST/J00369711).

Facilites: NuSTAR, XMM

\section{REFERENCES}

Arnaud, K. A. 1996, in ASP Conf. Ser. 101, Astronomical Data Analysis Software and Systems V, ed. G. H. Jacoby \& J. Barnes (San Francisco, CA: ASP), 17

Ballantyne, D. R., McDuffie, J. R., \& Rusin, J. S. 2011, ApJ, 734, 112

Ballantyne, D. R., Ross, R. R., \& Fabian, A. C. 2001, MNRAS, 327, 10

Ballantyne, D. R., Turner, N. J., \& Blaes, O. M. 2004, ApJ, 603, 436

Berti, E., \& Volonteri, M. 2008, ApJ, 684, 822

Bianchi, S., Guainazzi, M., Matt, G., Fonseca Bonilla, N., \& Ponti, G. 2009, A\&A, 495, 421

Blandford, R. D., \& Znajek, R. L. 1977, MNRAS, 179, 433

Blustin, A. J., Page, M. J., Fuerst, S. V., Branduardi-Raymont, G., \& Ashton, C. E. 2005, A\&A, 431, 111

Brenneman, L. 2013, Measuring the Angular Momentum of Supermassive Black Holes (New York: Springer)

Brenneman, L. W., \& Reynolds, C. S. 2006, ApJ, 652, 1028

Brenneman, L. W., Reynolds, C. S., Nowak, M. A., et al. 2011, ApJ, 736, 103

Brenneman, L. W., Risaliti, G., Elvis, M., \& Nardini, E. 2013, MNRAS, 429, 2662

Cackett, E. M., Fabian, A. C., Zogbhi, A., et al. 2013, ApJL, 764, L9

Connolly, S. D., McHardy, I. M., \& Dwelly, T. 2014, MNRAS, 440, 3503

Cowperthwaite, P. S., \& Reynolds, C. S. 2012, ApJL, 752, L21

Dadina, M. 2007, A\&A, 461, 1209

Dai, X., Kochanek, C. S., Chartas, G., et al. 2010, ApJ, 709, 278

Dauser, T., Garcia, J., Wilms, J., et al. 2013, MNRAS, 430, 1694

Dauser, T., Wilms, J., Reynolds, C. S., \& Brenneman, L. W. 2010, MNRAS, 409, 1534

de La Calle Pérez, I., Longinotti, A. L., Guainazzi, M., et al. 2010, A\&A, 524, A50

De Marco, B., Ponti, G., Cappi, M., et al. 2013, MNRAS, 431, 2441

Done, C., Jin, C., Middleton, M., \& Ward, M. 2013, MNRAS, 434, 1955

Dovciak, M., Matt, G., Bianchi, S., et al. 2013, arXiv:1306.2331

Dubois, Y., Volonteri, M., \& Silk, J. 2013, MNRAS, 440, 1590

Emmanoulopoulos, D., McHardy, I. M., \& Papadakis, I. E. 2011, MNRAS, 416, L94

Fabian, A. C., Kara, E., Walton, D. J., et al. 2013, MNRAS, 429, 2917

Fabian, A. C., Rees, M. J., Stella, L., \& White, N. E. 1989, MNRAS, 238, 729

Fabian, A. C., Zoghbi, A., Ross, R. R., et al. 2009, Natur, 459, 540

Gallo, L. C., Fabian, A. C., Grupe, D., et al. 2013, MNRAS, 428, 1191

García, J., \& Kallman, T. R. 2010, ApJ, 718, 695

George, I. M., \& Fabian, A. C. 1991, MNRAS, 249, 352

Harrison, F. A., Craig, W. W., Christensen, F. E., et al. 2013, ApJ, 770, 103

Jansen, F., Lumb, D., Altieri, B., et al. 2001, A\&A, 365, L1

Kallman, T., \& Bautista, M. 2001, ApJS, 133, 221

Kara, E., Fabian, A. C., Cackett, E. M., et al. 2013, MNRAS, 434, 1129

Kara, E., Zoghbi, A., Marinucci, A., et al. 2014, MNRAS, submitted

Kaspi, S., Maoz, D., Netzer, H., et al. 2005, ApJ, 629, 61

King, A. L., Miller, J. M., Gültekin, K., et al. 2013, ApJ, 771, 84

King, A. L., Walton, D. J., Miller, J. M., et al. 2014, ApJL, 784, L2

Kotov, O., Churazov, E., \& Gilfanov, M. 2001, MNRAS, 327, 799

Laor, A. 1991, ApJ, 376, 90

Legg, E., Miller, L., Turner, T. J., et al. 2012, ApJ, 760, 73
Lohfink, A. M., Reynolds, C. S., Jorstad, S. G., et al. 2013, ApJ, 772, 83

Lohfink, A. M., Reynolds, C. S., Miller, J. M., et al. 2012, ApJ, 758, 67

Magdziarz, P., \& Zdziarski, A. A. 1995, MNRAS, 273, 837

Maiolino, R., Risaliti, G., Salvati, M., et al. 2010, A\&A, 517, A47

Marinucci, A., Matt, G., Kara, E., et al. 2014a, MNRAS, 440, 2347

Marinucci, A., Matt, G., Miniutti, G., et al. 2014b, arXiv:1404.3561

Miller, J. M. 2007, ARA\&A, 45, 441

Miller, J. M., Miller, M. C., \& Reynolds, C. S. 2011, ApJL, 731, L5

Miller, J. M., Parker, M. L., Fuerst, F., et al. 2013, ApJL, 775, L45

Miller, J. M., Pooley, G. G., Fabian, A. C., et al. 2012, ApJ, 757, 11

Miller, L., \& Turner, T. J. 2013, ApJL, 773, L5

Miller, L., Turner, T. J., \& Reeves, J. N. 2008, A\&A, 483, 437

Miller, L., Turner, T. J., \& Reeves, J. N. 2009, MNRAS, 399, L69

Miller, L., Turner, T. J., Reeves, J. N., \& Braito, V. 2010, MNRAS, 408, 1928

Miniutti, G., \& Fabian, A. C. 2004, MNRAS, 349, 1435

Miniutti, G., Panessa, F., de Rosa, A., et al. 2009, MNRAS, 398, 255

Nandra, K., Barret, D., Barcons, X., et al. 2013, arXiv:1306.2307

Nandra, K., O’Neill, P. M., George, I. M., \& Reeves, J. N. 2007, MNRAS, 382,194

Nandra, K., \& Pounds, K. A. 1994, MNRAS, 268, 405

Nardini, E., Fabian, A. C., Reis, R. C., \& Walton, D. J. 2011, MNRAS, 410, 1251

Nardini, E., Fabian, A. C., \& Walton, D. J. 2012, MNRAS, 423, 3299

Nayakshin, S., Kazanas, D., \& Kallman, T. R. 2000, ApJ, 537, 833

Parker, M. L., Marinucci, A., Brenneman, L., et al. 2014a, MNRAS, 437, 721

Parker, M. L., Walton, D. J., Fabian, A. C., \& Risaliti, G. 2014b, arXiv:1404.2611

Parker, M. L., Wilkins, D. R., Fabian, A. C., et al. 2014c, MNRAS, submitted

Perola, G. C., Matt, G., Cappi, M., et al. 2002, A\&A, 389, 802

Reis, R. C., Fabian, A. C., Reynolds, C. S., et al. 2012a, ApJ, 745, 93

Reis, R. C., Miller, J. M., Reynolds, M. T., Fabian, A. C., \& Walton, D. J. 2012b, ApJ, 751, 34

Reis, R. C., Reynolds, M. T., Miller, J. M., \& Walton, D. J. 2014, Natur, 507, 207

Reynolds, C. S. 2013, SSRv

Reynolds, C. S., \& Fabian, A. C. 2008, ApJ, 675, 1048

Risaliti, G., Bianchi, S., Matt, G., et al. 2005a, ApJL, 630, L129

Risaliti, G., Elvis, M., Fabbiano, G., Baldi, A., \& Zezas, A. 2005b, ApJL, 623, L93

Risaliti, G., Elvis, M., \& Nicastro, F. 2002, ApJ, 571, 234

Risaliti, G., Harrison, F. A., Madsen, K. K., et al. 2013, Natur, 494, 449

Risaliti, G., Miniutti, G., Elvis, M., et al. 2009, ApJ, 696, 160

Rivers, E., Markowitz, A., \& Rothschild, R. 2013, ApJ, 772, 114

Ross, R. R., \& Fabian, A. C. 2005, MNRAS, 358, 211

Russell, D. M., Gallo, E., \& Fender, R. P. 2013, MNRAS, 431, 405

Schulz, H., Komossa, S., Schmitz, C., \& Mücke, A. 1999, A\&A, 346, 764

Sim, S. A., Proga, D., Miller, L., Long, K. S., \& Turner, T. J. 2010, MNRAS, 408, 1396

Steiner, J. F., McClintock, J. E., \& Narayan, R. 2013, ApJ, 762, 104

Steiner, J. F., Reis, R. C., Fabian, A. C., et al. 2012, MNRAS, 427, 2552

Strüder, L., Briel, U., Dennerl, K., et al. 2001, A\&A, 365, L18

Takahashi, T., Mitsuda, K., Kelley, R., et al. 2012, Proc. SPIE, 8443, 84431 Z

Tatum, M. M., Turner, T. J., Miller, L., \& Reeves, J. N. 2013, ApJ, 762, 80

Tomsick, J. A., Nowak, M. A., Parker, M., et al. 2014, ApJ, 780, 78

Turner, M. J. L., Abbey, A., Arnaud, M., et al. 2001, A\&A, 365, L27

Walton, D. J., Fuerst, F., Harrison, F., et al. 2013a, ApJ, 779, 148

Walton, D. J., Harrison, F. A., Grefenstette, B. W., et al. 2014, arXiv:1402.2992

Walton, D. J., Nardini, E., Fabian, A. C., Gallo, L. C., \& Reis, R. C. 2013b, MNRAS, 428, 2901

Walton, D. J., Reis, R. C., Cackett, E. M., Fabian, A. C., \& Miller, J. M. 2012, MNRAS, 422, 2510

Walton, D. J., Reis, R. C., \& Fabian, A. C. 2010, MNRAS, 408, 601

Walton, D. J., Zoghbi, A., Cackett, E. M., et al. 2013c, ApJL, 777, L23

Wang, J., Fabbiano, G., Elvis, M., et al. 2009, ApJ, 694, 718

Wilkins, D. R., \& Fabian, A. C. 2012, MNRAS, 424, 1284

Zhang, S. N., Cui, W., \& Chen, W. 1997, ApJL, 482, L155

Zoghbi, A., Fabian, A. C., Reynolds, C. S., \& Cackett, E. M. 2012, MNRAS, 422,129

Zoghbi, A., Fabian, A. C., Uttley, P., et al. 2010, MNRAS, 401, 2419

Zoghbi, A., Reynolds, C., Cackett, E. M., et al. 2013, ApJ, 767, 121 